\title{
Systematic astrometric observations of Proteus ${ }^{\star \star \star}$
}

\author{
R. Vieira Martins ${ }^{1,2}$, C. H. Veiga ${ }^{1}$, P. Bourget ${ }^{1}$, A. H. Andrei ${ }^{1,2}$, and P. Descamps ${ }^{3}$ \\ 1 Observatório Nacional, Rua General José Cristino 77, 20921-400 Rio de Janeiro, Brazil \\ e-mail: rvm@on.br \\ 2 Observatório do Valongo, Ladeira Pedro Antônio 43, 20080-090 Rio de Janeiro, Brazil \\ 3 Institut de Mécanique Céleste et de Calculs des Éphémérides, 77 avenue Denfert-Rochereau, 75014 Paris, France
}

Received 22 May 2003 / Accepted 14 May 2004

\begin{abstract}
Earth based observations of Proteus are made highly difficult by the magnitude difference with Neptune, and the closeness to the planet. To overcome these difficulties, observation campaigns using a Hg-mask coronograph developed for astrometry are being pursued at the Laboratório Nacional de Astrofísica, Brazil. The observations are made at visible light wavelengths with the $1.6 \mathrm{~m}$ reflector telescope. The large $4^{\prime} \times 4^{\prime}$ field of view of the camera makes it possible to image a sufficient number of background stars that are used to carry out the astrometric analysis of these data.

The first results of the campaigns are presented in this paper. They include 45 independent observations made over 2 nights in 2002. The positions are referred to the Hipparcos/Tycho2 frame. The mean offsets relative to the JPL ephemeris are $-0{ }^{\prime} 083\left(\sigma=0{ }^{\prime} 148\right)$ in the $x=\Delta \alpha \cos \delta$ direction, and $-0{ }^{\prime} 051\left(\sigma=0{ }^{\prime} 115\right)$ in the $y=\Delta \delta$ direction. Additional astrometric data need to be collected in order to investigate further the possible offset of Proteus with respect to the Voyager ephemerides. For the sake of completeness the early 2000 and 2001 campaigns, although less accurate, are also discussed.
\end{abstract}

Key words. astrometry - planets and satellites: individual: Proteus

\section{Introduction}

The Neptune satellite Proteus was discovered in 1989, when the spacecraft Voyager 2 imaged the inner system of Neptune (Smith et al. 1989). Using these observations, Owen et al. (1991) determined the satellite orbital elements. In 1991, Colas \& Buil (1992) using the ESO $2.2 \mathrm{~m}$ telescope made the first Earth-based observation of Proteus. By adding several frames, they obtained 8 positions of Proteus within a $70^{\prime \prime} \times 70^{\prime \prime}$ field. In 1995, Roddier et al. (1997) obtained the first groundbased adaptive optics observations of Proteus, using a system mounted on the CFH 3.6 m telescope. They obtained $4 K$-band images from $36^{\prime \prime} \times 36^{\prime \prime}$ fields on 1 night. Using HST, in 1995, Pascu et al. (1999), acquired 39 PC2 images of Proteus in a 2 days shift. The field was small and the frames were calibrated using Neptune and Triton positions, as well as with the Uranus system images. Finally, on 1998, Dumas et al. (2002) observed Proteus with the HST NICMOS near-infrared camera. They obtained 11 images in the $K$ band over two nights, in a field of $19^{\prime \prime} \times 19^{\prime \prime}$.

The positions measured by Colas \& Buil compare well to the orbit calculated by Owen et al., leading to rms orbital

\footnotetext{
* The catalog is only available in electronic form at the CDS via anonymous ftp to cdsarc.u-strasbg.fr (130.79.128.5) or via http: //cdsweb.u-strasbg.fr/cgi-bin/qcat?J/A+A/425/1107 $\star \star$ Based on observations made at Laboratório Nacional de Astrofísica/MCT-Itajubá-Brazil.
}

residuals of 0.'08. Likewise, Rodier et al., Pascu et al. and Dumas et al., reported that Proteus could be seen at the places predicted from the Voyager data.

The LNA program of systematic astrometric observations of planetary satellites started on 1982. Concerning the Neptunian satellites Triton and Nereid, results were published in Veiga et al. (1996), Veiga \& Vieira Martins (1996, 1998), and Veiga et al. (1999). In 2000, to observe faint satellites close to the much brighter planets, a variable-diameter occulting disk coronagraph was constructed (Bourget et al. 2001). It has made it possible to observe Proteus.

The initial observations of the satellite were done on that same year, 2000, and on the following, 2001. These initial trials led the way to the more extended 2002 campaign, whose results are presented here. In Sect. 2, the observations and the reduction are described. The comparison between the observational and the theoretical positions are discussed in Sect. 3. The conclusions are summarized in Sect. 4.

\section{Observations and reductions}

All the observations here reported were made at the Cassegrain focus of the $1.6 \mathrm{~m}$ Ritchey-Chretien reflector of the LNA Laboratório Nacional de Astrofísica, in Brazil, (geographical longitude: $3^{\mathrm{h}} 02^{\mathrm{m}} 19^{\mathrm{s}}$, latitude: $-22^{\circ} 32^{\prime} 04^{\prime \prime}$, and altitude: $1872 \mathrm{~m}$ ). The focal length of the Cassegrain combination is $15.8 \mathrm{~m}$, which results in a plate scale of $13^{\prime \prime} / \mathrm{mm}$ in the focal 


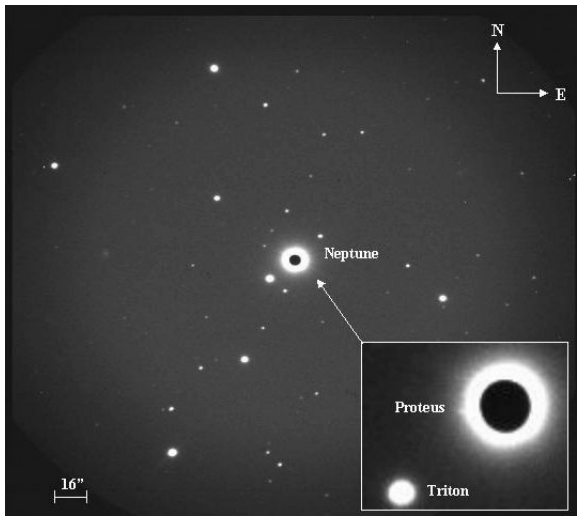

Fig. 1. Frame of the Neptune system as observed on 08/09/2002, at $0^{\mathrm{h}} 4^{\mathrm{m}} 46^{\mathrm{s}}$ UTC. The satellite Proteus is just to the right of its label and on the edge of the scattered light of Neptune. The CCD has $1024 \times$ 1024 square pixels with sides $22 \mu \mathrm{m}$. The exposure time was $20^{\text {s }}$.

Table 1. General information about the observations. In the table, (date) is that of observation night, $(T)$ is the total time length of the observation in minutes, $(N)$ is the number of images, $(S)$ is the mean number of reference stars, $(C)$ is the dimension of the CCD in pixel, $(P)$ is the size of the pixel in $\mu \mathrm{m}$ and $(s)$ is the plate scale in arcsecond. For the CCD used in 2000 the pixel is binned from the original $9 \mu \mathrm{m} \times 9 \mu \mathrm{m}$ size.

\begin{tabular}{ccccccc}
\hline \hline Date & $T$ & $N$ & $S$ & $C$ & $P$ & $s$ \\
\hline $2002-08-08$ & 46 & 23 & 50 & $1024 \times 1024$ & $22 \times 22$ & 0.30 \\
$2002-08-10$ & 96 & 22 & 40 & $1024 \times 1024$ & $22 \times 22$ & 0.30 \\
\hline $2001-08-21$ & 111 & 15 & 20 & $1024 \times 1024$ & $22 \times 22$ & 0.30 \\
$2000-10-12$ & 60 & 4 & 12 & $1536 \times 1024$ & $18 \times 18$ & 0.25 \\
\hline
\end{tabular}

plane. A coronagraph with a variable-diameter occulting disk (Bourget et al. 2001) was used to shield the light of the planet. Its optics causes small changes in the plate scale as later discussed. To avoid the diffraction spikes from the secondary mirror bearing structure in the telescope focal plane, a mask with eight circular holes is placed on the entrance pupil.

The campaign comprised two nights, in which 45 observations of Proteus were taken. The general information about these observations is presented in the first two rows of Table 1. Additionally, the two bottom rows present general information concerning the preliminary 2000 and 2001 campaigns.

During the observations the diameter of the occulting disk was changed depending on the seeing conditions. For every frame the disk was re-centered onto the planet image, which is about $2^{\prime \prime}$ in diameter, in order to compensate for the motion of the planet and for any telescope drift. The disk centering procedure is accurate to better than $1^{\prime \prime}$. The exposure time was changed according to the planet-to-satellite distance and to the seeing conditions. The mean exposure time was around $20 \mathrm{~s}$. All observations were carried out when the satellite was near the maximum elongation, that is about $5^{\prime \prime}$ from the planet. An example frame is presented in Fig. 1. It well illustrates the difficulty that is involved in taking these observations.

Figure 2 presents the orbits of Proteus and Triton at the epoch of the observations, and the positions of the satellites in
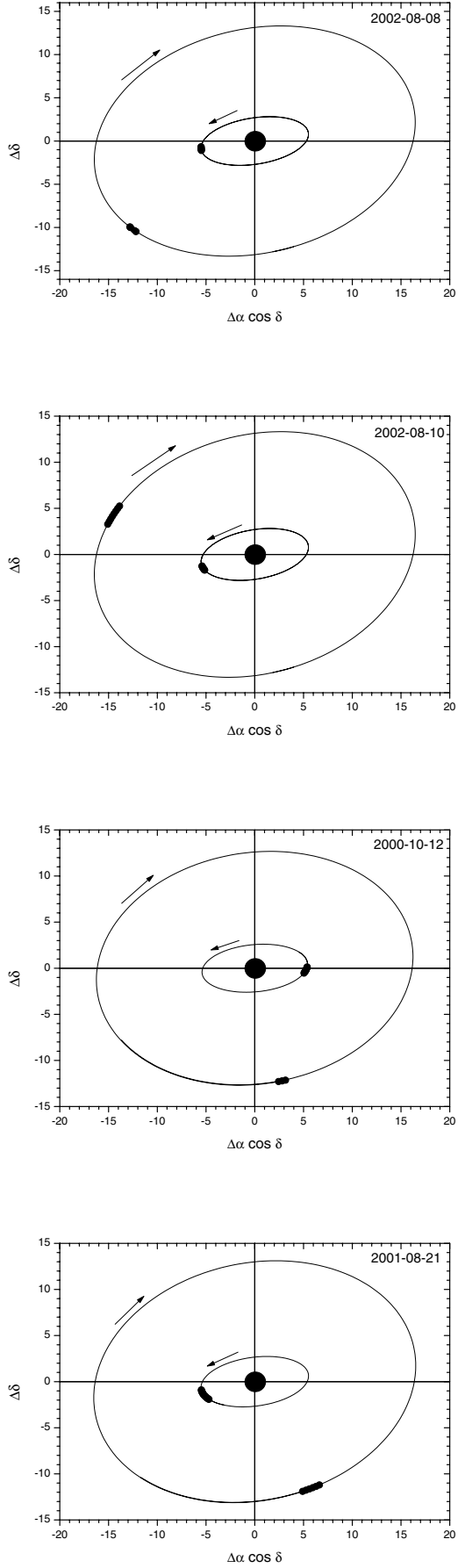

Fig. 2. Proteus and Triton in their orbits on each observation night. The initial 2000 and 2001 data are also shown for completeness.

their orbits during the corresponding night, both for the observational period and for the preliminary campaigns. It is worth noticing that for all nights, except for the 2000 one, the positions of Proteus in the orbit are almost the same.

All frames were measured with the ASTROL software (Colas 1996), which uses a centering algorithm based on a fit with a point spread function. The local sky background was removed by ASTROL, using a fit with a second order polynomial. This background removal is essential for avoiding systematic errors in the measurements of the center of Proteus. The center of Neptune cannot be measured since the mask is 


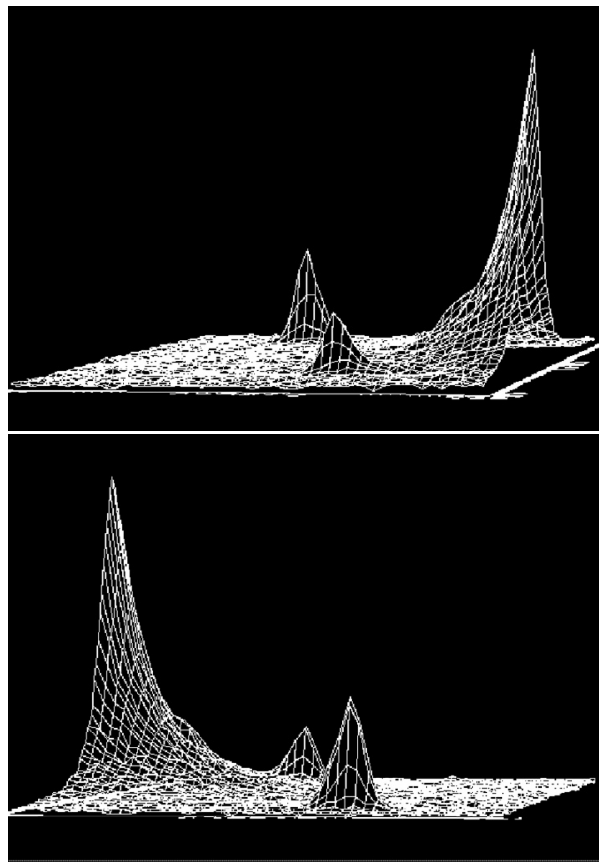

Fig. 3. The picture shows two 3D views of one Proteus observation. The tallest feature is Neptune's light. Proteus is seen as a separate structure on the lower slope of Neptune. Although smaller than the structures of the two field stars shown, the structure of Proteus is clearly higher than the noise background, and distinct from Neptune's scattered light distribution.

over the image of the planet. For the stars and for Triton, the centering errors are at the level of 0.01 pixel and for Proteus it is at the level of 0.4 pixel.

Two surface plots of the image of Proteus are shown in Fig. 3. The signature of the satellite is clearly seen on the slope of the scatttered light of the planet. To further verify the efficiency of ASTROL in this case, two tests were made, using the observation shown. In one test, the planet light was removed by a Gaussian fit, while in the other test its removal was by a second degree polynomial fit. Both tests were performed along three rows, centered on the ASTROL position for Proteus. After the removal of the light of the planet, the position of Proteus was found by adjusting a one-dimensional Gaussian. The signal to noise rate at the peak of the Gaussian is around 4. On averaging the six rows examined, the center of Proteus is found at $0 . ' 13$ from the ASTROL position (the differences range from 0. .'07 to $0^{\prime \prime} 28$ ). The obtained values are comparable to those obtained when making a similar Gaussian fit to the center of Triton, which is much brighter and not affected by the scattered light of the planet. Figure 4 gives a graphical representation of this process. In the Fig. $4 \mathrm{a}$, the counts are presented for some 17 pixels of a CCD line which cuts the Proteus image in the direction of the center of Neptune. The five pixels around the presumed Proteus center were removed to perform the sky fit. In the figure these pixels are inside the dashed rectangle. In Fig. 4b, the sky fit was subtracted from every pixel count and the resulting count distribution was fitted by a Gaussian curve. It can be observed that the image of Proteus is clearly above the corrected sky level.
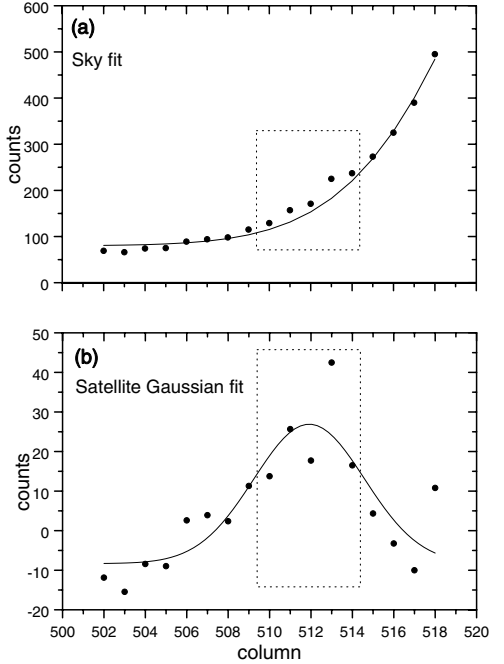

Fig. 4. Graphical representation of the removal of the scattered light of the planet for one CCD line. In a), the sky is fitted for all line pixels except for the five inside the rectangle. In b), the sky fit was subtracted from every pixel count and the resulting points were fitted with a Gaussian curve. Observe that the sky level is at about -10 counts and that the new image of Proteus is above this sky level.

The coronagraph optical system imposes a correction of the third degree for the astrometric calibration of the observed field. This calibration was made using a third degree polynomial (10-constant model) to map the tangential plane projection of the sky onto the frame. The equatorial coordinates of the field stars were obtained from the USNO-A2.0 catalog, as locally corrected by the Tycho 2 positions (Assafin et al. 2001) at the epoch of the observation. The local corrections were calculated in 1 square degree regions, within which the A2.0 showed the expected coherency (i.e., no correction parameter at the 0.1 level except to those for linear transformation). On this basis, the stars presenting residuals larger than $0 . ' 6$ were rejected. This threshold corresponds to 3 times the catalog errors for a single entry. The standard deviation of the residuals for the stars effectively employed in the reduction was at 0 . $^{\prime} 15$.

As shown in Table 1, for the 2000 opening campaign the fields were small and so the number of reference stars was smaller than 15. As a consequence, first and second degree polynomials were tried for the astrometry in those frames. Both fits gave similar results. Thus, the most conservative approach was adopted and the first degree polynomial was retained for the astrometric calibration.

\section{Comparison with the theoretical positions}

The observations were compared with the positions from the JPL ephemeris (Jacobson 2002). The calculated positions for Proteus are based on a precessional ellipse computed by Owen et al. (1991) from the Voyager 2 measurements of the Neptunian system. For the definitive 2002 campaign, the observed Proteus positions for which the observed residuals minus the calculated ones were larger than $1^{\prime \prime}$, and/or the observations in which there were less than 15 reference stars were discarded. The first criterion removes determinations worse 

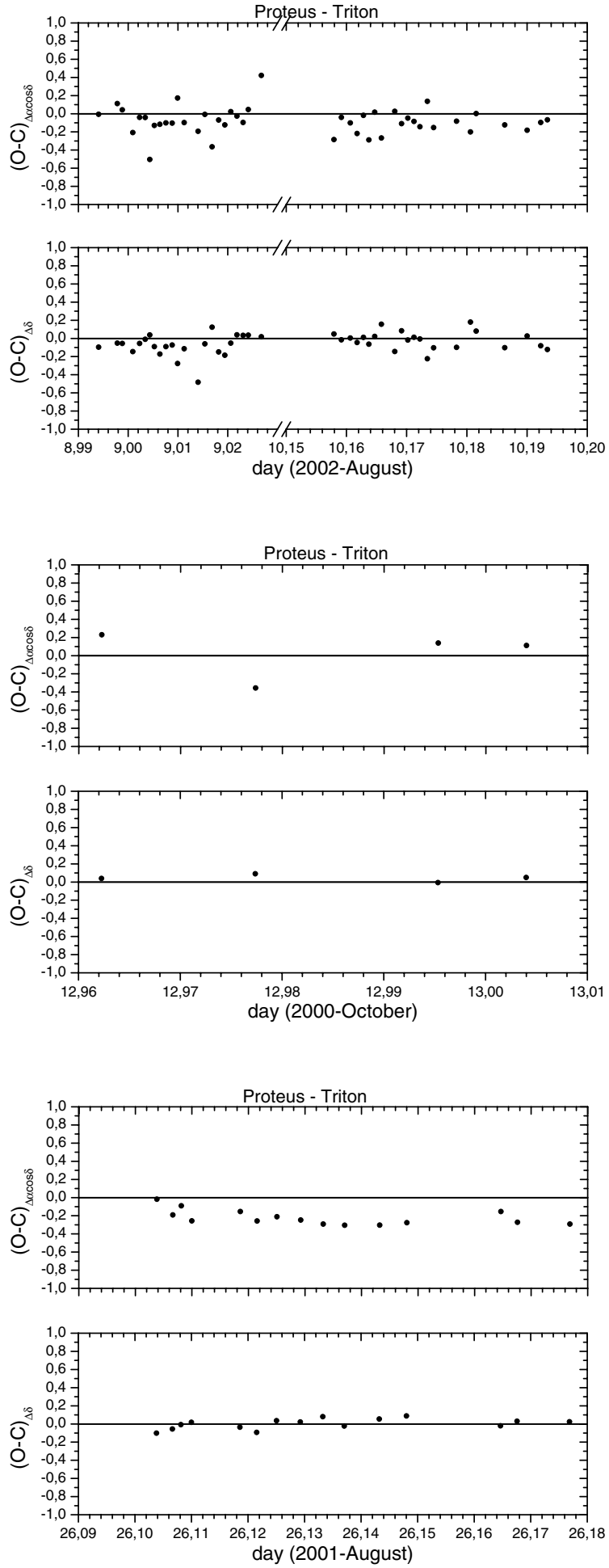

Fig. 5. Observed minus calculated residuals for Proteus relative to Triton. The $(\mathrm{O}-\mathrm{C})$ are given in arcsecond.

than 3 times the combined centering error for Proteus and the standard deviation from the reference stars. The second criterion is derived from the analysis of the initial trials in the 2000 campaign. For the initial 2000 and 2001 campaigns, the same criteria are enforced here, except for the 15 stars threshold in the 2000 campaign.
Table 2. Observed minus calculated statistics for Proteus-Triton (P-T), Triton and Proteus in arcseconds. The meaning of the symbols is: $x=\Delta \alpha \cos \delta, y=\Delta \delta, \sigma$ for the standard deviation and $N$ for the number of frames. There are 44 observations for Triton and Proteus and 45 for Proteus-Triton because one observation for the satellites has $(\mathrm{O}-\mathrm{C})>1^{\prime \prime}$ and $(\mathrm{O}-\mathrm{C})<1^{\prime \prime}$ for their difference. The statistics for 2000 and 2001 are also given.

\begin{tabular}{ccccccc}
\hline \hline P-T & Date & $\bar{x}$ & $\bar{y}$ & $\sigma_{x}$ & $\sigma_{y}$ & $N$ \\
\hline & $2002-08-08$ & -0.062 & -0.082 & 0.178 & 0.125 & 23 \\
& $2002-08-10$ & -0.106 & -0.018 & 0.108 & 0.097 & 22 \\
All & & -0.083 & -0.051 & 0.148 & 0.115 & 45 \\
\hline \multirow{6}{*}{ Triton } & 2001-08-21 & -0.222 & 0.001 & 0.085 & 0.057 & 15 \\
& 2000-10-12 & 0.031 & 0.043 & 0.263 & 0.040 & 4 \\
\hline & Date & $\bar{\alpha}$ & $\bar{\delta}$ & $\sigma_{\alpha}$ & $\sigma_{\delta}$ & $N$ \\
\hline \multirow{4}{*}{ All } & $2002-08-08$ & -0.024 & -0.010 & 0.019 & 0.029 & 23 \\
& $2002-08-10$ & -0.071 & 0.005 & 0.073 & 0.084 & 21 \\
& & -0.046 & 0.003 & 0.057 & 0.062 & 44 \\
\hline Proteus & $2001-08-21$ & 0.138 & 0.187 & 0.251 & 0.153 & 15 \\
& $2000-10-12$ & 0.030 & -0.010 & 0.145 & 0.027 & 4 \\
\hline \multirow{4}{*}{ All } & Date & $\bar{\alpha}$ & $\bar{\delta}$ & $\sigma_{\alpha}$ & $\sigma_{\delta}$ & $N$ \\
\hline \multirow{6}{*}{} & $2002-08-08$ & -0.098 & -0.071 & 0.190 & 0.126 & 23 \\
& $2002-08-10$ & -0.173 & -0.024 & 0.134 & 0.118 & 21 \\
& & -0.134 & 0.032 & 0.169 & 0.127 & 44 \\
\hline & $2001-08-21$ & -0.096 & 0.188 & 0.313 & 0.126 & 15 \\
& $2000-10-12$ & 0.053 & 0.033 & 0.134 & 0.066 & 4 \\
\hline
\end{tabular}

Figure 5 presents the observed minus calculated residuals for Proteus relative to Triton, and the corresponding statistics are found in Table 2. The right ascension and declination residuals for Triton are small, i.e., the results comply well with the theoretical values indicating the correctness of the present state of observations and data reduction. The residuals for Proteus reveal a larger dispersal, due to the difficulty in the centering process. If this is taken into account, there is a suggestion that the fit to the ephemeris in the $x$ direction is less good. From this data, there is an offset in the observed positions in the negative $x$ direction points of maximum elongation. This result however must be further verified since the effect of the light of the planet is more asymmetric in that direction, and consequently the polynomial that fits the sky in the region cannot model the effect well. Notice that, for all campaigns, the spread of the residuals is large in the $x$ than in the y direction.

Some cautionary notes on the shortcomings of the 2000 and 2001 initial campaigns will be added. Regarding the year 2000 , there are only 4 observations and so it is difficult to draw firm conclusions from them. The dispersion is probably due to bad conditions of the observations themselves and also to the poorer astrometric calibration. For the year 2001 the offset of the residuals in the $x$ direction is particularly large. Still, this is probably not due to the centering algorithm used, since another algorithm (TASP) was used and the results are very similar. A more likely explanation for the large mean offset is related to the small number of field stars used (see Table 1) in the astrometric calibration, which employed a third degree polynomial. 
This was checked by examining the residuals in right ascension and declination for Triton (see Table 2). These residuals present large offsets and spreading, suggesting that the bad result is due to the astrometric calibration.

\section{Conclusion}

The use of the Hg-mask coronograph made it possible to make astrometric observations of Proteus, near the maximum elongation. Initial campaigns to refine the modes of observation and data reduction were launched in 2000 and 2001. Although interesting because there had not been many observations of this kind, their results were weakened by the small number of frames obtained, their smaller sizes, and the small number of reference stars within. On the other hand, the results for the 2002 campaign benefit from the previous experience on all these points. Their results are coherent, given standard deviations of the residuals that agree with the theoretical values for the centering errors, for Proteus as well as for Triton. For the latter, the results comply with the JPL ephemeris. However for Proteus the comparison between the calculated and the observed orbits indicates a possible 0 '. $^{\prime}$ offset of the observed orbit in the negative right ascension direction near the maximal elongation positions. In view of this, we are planning to continue the astrometric observations of Proteus at Neptune opposition.

Acknowledgements. RVM wishes to thank the staff of IMCCE where, during his stay form November to December of 2002, this research was concluded. The authors thank O. L. Chaves, F. Vachier and R. Campos for their help in the observations. C. H. Veiga would like to thank the State of Rio de Janeiro Science FoundationFAPERJ for providing computational facilities for this project.

\section{References}

Assafin, M., Andrei, A. H., Vieira Martins, R., et al. 2001, ApJ, 552, 380

Bourget, P., Veiga, C. H., \& Vieira Martins, R. 2001, PASP, 113, 436

Colas, F. 1996, in Proc. of the Phesat 95 Workshop, Supp. Ann. de Phys., 21, 113

Colas, F., \& Buil, C. 1992, A\&A, 262, L13

Dumas, C., Terrile, R. J., Smith, B. A., \& Schneider, G. 2002, AJ, 123, 1776

Jacobson, R. A. 2002, personal communication

Owen, W. M., Vaughan, R. M., \& Synott, S. P. 1991, AJ, 101, 1511

Pascu, D., Rohde, J. R., Seidelmann, P. K., et al. 1999, BAAS, 31, 1229

Roddier, F., Roddier, C., Brahic, A., et al. 1997, Planet. Space Sci., 45, 1031

Smith, B. A., Soderblom, L. A., Bamfield, D., et al. 1989, Science, 246,1422

Veiga, C. H., \& Vieira Martins, R. 1996, A\&AS, 120, 107

Veiga, C. H., \& Vieira Martins, R. 1998, A\&AS, 131, 291

Veiga, C. H., Vieira Martins, R., \& Le Guyader, Cl. 1999, A\&AS, 136, 445

Veiga, C. H., Vieira Martins, R., Le Guyader, Cl., \& Assafin, M. 1996, A\&AS, 115, 319 